\title{
On the relationship between metrics to compare greenhouse gases - the case of IGTP, GWP and SGTP
}

\author{
C. Azar and D. J. A. Johansson \\ Physical Resource Theory, Chalmers University of Technology, 41296 Gothenburg, Sweden \\ Correspondence to: D. J. A. Johansson (daniel.johansson@chalmers.se) \\ Received: 18 January 2012 - Published in Earth Syst. Dynam. Discuss.: 6 February 2012 \\ Revised: 19 September 2012 - Accepted: 8 October 2012 - Published: 2 November 2012
}

\begin{abstract}
Metrics for comparing greenhouse gases are analyzed, with a particular focus on the integrated temperature change potential (IGTP) following a call from IPCC to investigate this metric. It is shown that the global warming potential (GWP) and IGTP are asymptotically equal when the time horizon approaches infinity when standard assumptions about a constant background atmosphere are used. The difference between IGTP and GWP is estimated for different greenhouse gases using an upwelling diffusion energy balance model with different assumptions on the climate sensitivity and the parameterization governing the rate of ocean heat uptake. It is found that GWP and IGTP differ by some $10 \%$ for $\mathrm{CH}_{4}$ (for a time horizon of less than $500 \mathrm{yr}$ ), and that the relative difference between GWP and IGTP is less for gases with a longer atmospheric life time. Further, it is found that the relative difference between IGTP and GWP increases with increasing rates of ocean heat uptake and increasing climate sensitivity since these changes increase the inertia of the climate system. Furthermore, it is shown that IGTP is equivalent to the sustained global temperature change potential (SGTP) under standard assumptions when estimating GWPs. We conclude that while it matters little for abatement policy whether IGTP, SGTP or GWP is used when making tradeoffs, it is more important to decide whether society should use a metric based on time integrated effects such as GWP, a "snapshot metric" as GTP, or metrics where both economics and physical considerations are taken into account. Of equal importance is the question of how to choose the time horizon, regardless of the chosen metric. For both these overall questions, value judgments are needed.
\end{abstract}

\section{Introduction}

A range of metrics for comparing and aggregating the climate effect of different greenhouse gases has been proposed. The most widely applied metric is the global warming potential (GWP). When estimating the GWP, the radiative forcing from a pulse emission, say $1 \mathrm{~kg}$ of gas $X$ at time $t=0$, is integrated until an arbitrary time horizon $H$, and divided by the result of an equivalent integration for the reference gas, usually $\mathrm{CO}_{2}$. GWP is the standard option when comparing emissions of different greenhouse gases, e.g. in the Kyoto protocol. It was originally developed by Rodhe (1990), Shine et al. (1990) and Lashof and Ahuja (1990). See Forster et al. (2007) for the estimates of GWP values developed for IPCC's Fourth Assessment Report.

An alternative to the GWP that has received more attention recently is the global temperature change potential (GTP). When estimating the GTP the temperature response at time $t=H$ from a pulse emission of gas $X$ at time $t=0$ is divided by the equivalent temperature response for a reference gas, usually $\mathrm{CO}_{2}$. It should be noted that GTP only considers the temperature response at $t=H$. Thus, it is different from GWP in the sense that GWP is an integrative measure (of the radiative forcing contribution over the entire period). GTP was initially proposed by Shine et al. (2005).

There is a renewed interest among researchers and policy makers to investigate the performance of other alternative metrics. A special meeting on metrics for comparing greenhouse gases was organized by the IPCC in Oslo in 2009 (IPCC, 2009). In its recommendations to the scientific community regarding research needs, IPCC (2009) writes that researchers should "develop new and refined metrics", e.g. a metric based on "the integral of the temperature change" 
following a pulse emission of a gas $X$ compared to "the integral of the temperature change" following a pulse emission of $\mathrm{CO}_{2}$.

Such a measure has been discussed previously by, for instance, Fisher et al. (1990), Rotmans and den Elzen (1992), Shine et al. (2005, p. 298), IPCC (2009), Gillet and Matthews (2010) and Peters et al. (2011). We here refer to this metric as the Integrated Global Temperature change Potential (IGTP). Note that it is not GTP that is integrated, but the temperature response from gas $X$ divided by the integrated temperature response from the reference gas.

In addition to GWP, GTP and IGTP, Shine et al. (2005) proposed a Sustained Global Temperature change Potential $(S G T P)$ as the temperature response at time $t=H$ of a gas $X$ emitted with a constant (sustained) rate $\left(1 \mathrm{~kg} \mathrm{yr}^{-1}\right)$ divided by the temperature response at time $t=H$ following sustained emissions of $\mathrm{CO}_{2}\left(1 \mathrm{~kg} \mathrm{yr}^{-1}\right)$. Fisher et al. (1990) also estimated halocarbon global warming potentials, i.e. a metric for the integrated warming effect of various halocarbons compared to CFC 11, that was defined in the same way as SGTP with the main difference that infinite time horizons were used. They could use infinite time horizons since halocarbons have exponential decay rates (which ensure convergence of the relevant integrals).

The purpose of our paper is to analyze the properties of IGTP and demonstrate its relationship with GWP and SGTP. Relations between IGTP, SGTP and GWP have been noted in the literature. O'Neill (2000) showed that IGTP and GWP are equivalent when the time horizon approaches infinity under special conditions. Shine et al. (2005) find a "near equivalence" between SGTP and GWP. This implies that IGTP and GWP are also near equivalent since IGTP and SGTP, as will be shown in this paper, are identical under "standard" assumptions when calculating GWP values, i.e. constant radiative efficiencies (defined as the radiative forcing in $\mathrm{W} \mathrm{m}^{-2} \mathrm{~kg}^{-1}$ ) and constant atmospheric adjustment times for the gases compared. Sarofim (2012) analyzed SGTP values for methane under the assumption of varying background concentrations. Further, Fisher et al. (1990) observe numerically that steady state values for SGTP (or HGWP in their terminology) are closely related to IGTP.

Recently, Peters et al. (2011) observed a close relationship between IGTP and GWP. In their paper, they conclude that "further modeling would be required to confirm these observations". In our paper, we use an upwelling diffusion energy balance model instead of the impulse response function approach they take to estimate IGTP. In addition, we carry out a more systematic sensitivity analysis with respect to the climate sensitivity and vertical heat diffusivity.

In Sect. 2, we present our model. The results are presented in Sect. 3 which also contains our sensitivity analysis. In Sect. 4 we explain our results. Conclusions are given in Sect. 5. In the appendices, we formally show that IGTP and SGTP are equivalent measures (under standard assumptions about constant atmospheric adjustment times and constant radiative efficiencies), and that IGTP and GWP are asymptotically equal as the time horizon approaches infinity.

\section{Method}

In this section, we present the method used to calculate IGTP (and GWP). IGTP is defined as

$$
\operatorname{IGTP}(H)=\int_{0}^{H} T_{\mathrm{P} x}(t) \mathrm{d} t / \int_{0}^{H} T_{\mathrm{P}, \mathrm{CO}_{2}}(t) \mathrm{d} t
$$

where $T_{\mathrm{P} x}(t)$ is the temperature response at time $t$ from a pulse emission of $1 \mathrm{~kg}$ of gas $X$ at time 0 (and similarly for the temperature response for a pulse emission of $1 \mathrm{~kg}$ of $\mathrm{CO}_{2}$ ). In Appendix A we show that IGTP is identical to SGTP (under standard assumptions when calculating GWPs). Thus all results presented in this paper that hold for the relationship between IGTP and GWP also hold for the relationship between SGTP and GWP (under the assumption of constant atmospheric adjustment times and constant radiative efficiencies).

Further, GWP is defined as

$$
\mathrm{GWP}_{x}=\frac{\int_{0}^{H} C_{\mathrm{P} x}(t) F_{x} \mathrm{~d} t}{\int_{0}^{H} C_{\mathrm{P}, \mathrm{CO}_{2}}(t) F_{\mathrm{CO}_{2}} \mathrm{~d} t}
$$

where $C_{\mathrm{P} x}(t)$ is the change in mass of greenhouse gas $X$ in the atmosphere at time $t$ following a pulse emission (of $1 \mathrm{~kg}$ ) at time $t=0$, and $F_{x}$ is the radiative efficiency per $\mathrm{kg}$ of gas $X$ in the atmosphere. We assume, as stated earlier, that the radiative efficiency and the atmospheric adjustment times are constant, which is standard when estimating GWP.

In order to estimate IGTP numerically, we use an Upwelling-Diffusion Energy Balance Model (UDEBM) developed in Johansson (2011). In the UDEBM the surface of the globe is divided into one fraction for the ocean (and the troposphere above it) and one fraction for the land surface (and troposphere above it). The equilibrium temperature change is set to be $30 \%$ higher for the surface air over land than over the oceans (see Raper et al., 2001 and Meinshausen et al., 2011a). The marine surface air temperature increase is assumed to be $30 \%$ higher than the ocean surface temperature increase due to retreating sea ice cover (see Raper et al., 2001 and Meinshausen et al., 2011a). The temperature of the water that down wells in the polar regions is assumed to increase by only a fifth of the increase in the global average sea surface temperature. The heat capacity of the land fraction is set to zero. All these model assumptions are basically standard in UDEBMs and similar models, see Raper et al. (2001), Shine et al. (2005), Meinshausen et al. (2011a), Baker and Roe (2009), and Olivié and Stuber (2010). 
Table 1. IGTP estimates for $\mathrm{CH}_{4}, \mathrm{~N}_{2} \mathrm{O}$ and $\mathrm{SF}_{6}$ (with GWP values in brackets).

\begin{tabular}{lccc}
\hline Time horizon & $\mathrm{CH}_{4}$ & $\mathrm{~N}_{2} \mathrm{O}$ & $\mathrm{SF}_{6}$ \\
\hline 20 & $77(72)$ & $284(289)$ & $15800(16200)$ \\
100 & $29(25)$ & $301(298)$ & $22100(22800)$ \\
500 & $8(8)$ & $162(153)$ & $32000(32600)$ \\
\hline
\end{tabular}

In the standard set up, climate sensitivity is set to $3 \mathrm{~K}$ for a doubling of the atmospheric $\mathrm{CO}_{2}$ concentration in line with IPCC's best estimate (Solomon et al., 2007). The likely range for the climate sensitivity is according to IPCC $2-4.5 \mathrm{~K}$ for a doubling of the atmospheric $\mathrm{CO}_{2}$ concentration. We use this range in our sensitivity analysis. The ocean heat mixing in the UDEBM is determined by the vertical heat diffusivity and the upwelling rate. The upwelling rate is set to $4 \mathrm{~m} \mathrm{yr}^{-1}$ (see Raper et al., 2001; Johansson, 2011; Meinshausen et al., 2011a) and the base case diffusivity is set to $2 \mathrm{~cm}^{2} \mathrm{~s}^{-1}$. In order to emulate the ocean heat uptake and the surface temperature response in more complex models, a diffusivity in the range $0.5-5$ is often used in UDEBMs (see Raper et al., 2001; Johansson, 2011; Meinshausen et al., 2011a; Baker and Roe, 2009; Olivié and Stuber, 2010). In the sensitivity analysis, we set this parameter at 0.5 and $4 \mathrm{~cm}^{2} \mathrm{~s}^{-1}$ as alternatives to our base case assumption. In Appendix B, we demonstrate that our choice for these parameter values, in the base case as well as in the sensitivity analyses, is compatible with the measured global average surface temperature change over the past hundred years.

For the atmospheric adjustment times for $\mathrm{CH}_{4}, \mathrm{~N}_{2} \mathrm{O}, \mathrm{SF}_{6}$ and $\mathrm{CO}_{2}$, we use the assumptions that are used when estimated GWP in IPCC AR4, see Forster et al. (2007). The radiative efficiency measured per $\mathrm{kg}$ gas is also taken from Forster et al. (2007).

For forcings that are globally rather heterogenous (e.g. ozone, aerosols and contrails), an equal change in global mean radiative forcing gives a different global mean temperature response, i.e. the climate efficacies are not equal (see e.g. Hansen et al., 2005). It could be argued that under such conditions global warming potentials should be recalculated so that the climate efficacy of gas $X$ is taken into account. In our calculations, we have assumed that the climate efficacies are equal to one throughout the paper.

\section{Results: comparison of IGTP and GWP}

We start by presenting numerical estimates for the GWP and IGTP for $\mathrm{CH}_{4}, \mathrm{~N}_{2} \mathrm{O}$ and $\mathrm{SF}_{6}$ in Table 1.

It can be noted that the GWP and IGTP values are close (see also Azar and Johansson, 2012 and Peters et al., 2011). One may also note that the $100 \mathrm{yr}$ IGTP values for methane are slightly higher than its corresponding GWP values, whereas the opposite holds for $\mathrm{SF}_{6}$. We will return to

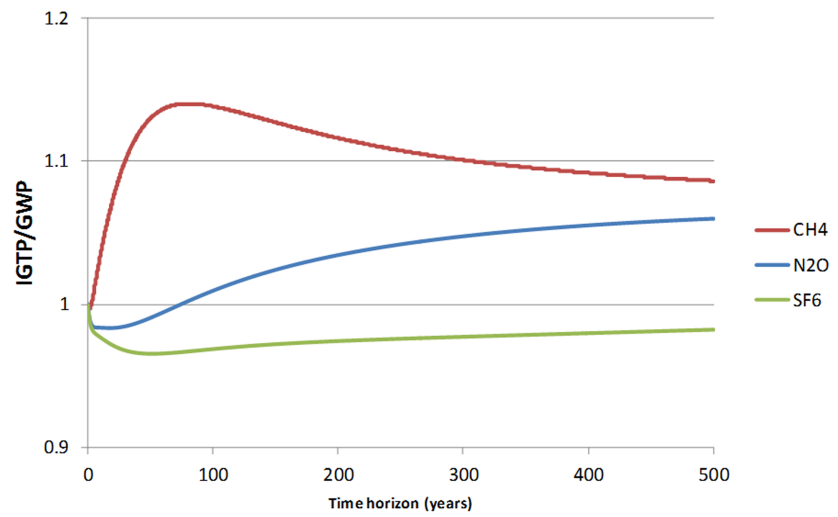

Fig. 1. IGTP/GWP ratio for $\mathrm{CH}_{4}, \mathrm{~N}_{2} \mathrm{O}$ and $\mathrm{SF}_{6}$ depending on the time horizon $H$ (in years).

this feature later on in the paper and see how it results from the fact that the gases have significantly shorter and longer perturbation life times than $\mathrm{CO}_{2}$.

The estimates for the IGTP/GWP ratios as a function of the time horizon, $\mathrm{H}$, for $\mathrm{CH}_{4}, \mathrm{~N}_{2} \mathrm{O}$ and $\mathrm{SF}_{6}$ with $\mathrm{CO}_{2}$ as the reference gas, can be seen in Fig. 1. The difference between GWP and IGTP is typically around or less than $10 \%$.

\section{Sensitivity analysis}

In this subsection, we focus on how the ratio between IGTP and GWP is affected by changes in the vertical heat diffusivity and climate sensitivity.

An increase in the vertical heat diffusivity results in the temperature increasing more slowly in response to changes in the radiative forcing, see e.g. Hansen et al. (1985) and Johansson (2011). This magnifies the difference between the equilibrium temperature change for a given forcing and the actual temperature change. As a consequence, the higher the heat diffusivity, the higher the inertia of the climate system, and the more IGTP will deviate in relative terms from GWP for all greenhouse gases (see Fig. 2). Decreasing the vertical heat diffusivity has the opposite effect, i.e. the IGTP/GWP ratio will for both short-lived and long-lived greenhouse become closer to unity.

Changing the climate sensitivity has a similar effect as changing the effective vertical heat diffusivity, since a larger climate sensitivity implies that the temperature responds, in relative terms, more slowly to changes in radiative forcing, see e.g. Hansen et al. (1985). This is shown for $\mathrm{CH}_{4}, \mathrm{~N}_{2} \mathrm{O}$ and $\mathrm{SF}_{6}$ in Fig. 3. Decreasing the climate sensitivity has the opposite effect, i.e. the IGTP/GWP ratio will for both shortlived and long-lived greenhouse become closer to unity.

Finally, in Appendix $\mathrm{C}$, we present numerical values for IGTP for $\mathrm{CH}_{4}, \mathrm{~N}_{2} \mathrm{O}$ and $\mathrm{SF}_{6}$ for different assumptions on the vertical heat diffusivity and the climate sensitivity. In most cases, IGTP values change modestly for even large changes in these two parameters. For example, a change in the time 


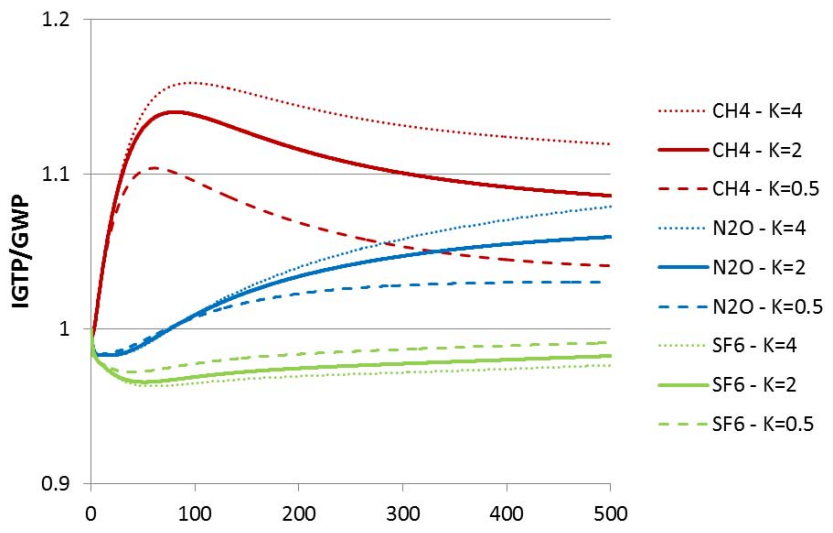

Fig. 2. IGTP/GWP ratio for $\mathrm{CH}_{4}, \mathrm{~N}_{2} \mathrm{O}$ and $\mathrm{SF}_{6}$ depending on effective vertical heat diffusivity. $K$, the vertical heat diffusivity, is given in $\mathrm{cm}^{2} \mathrm{~s}^{-1}$.

horizon (from 20 to $500 \mathrm{yr}$ ) changes IGTP values by a factor of two for $\mathrm{SF}_{6}$ and $\mathrm{N}_{2} \mathrm{O}$, and almost a factor of 10 for methane, but changes in the climate sensitivity and the heat uptake only affect the IGTP values by a few percent (in the absolute majority of cases).

\section{Interpreting the relationship between GWP and IGTP}

In this section we aim to explain and interpret the results presented in Sect. 3. The ratio of IGTP and GWP is given by dividing Eq. (1) with Eq. (2), i.e.

$$
\frac{\operatorname{IGTP}(H)}{\operatorname{GWP}(H)}=\frac{\int_{0}^{H} T_{\mathrm{P} x}(t) \mathrm{d} t}{\int_{0}^{H} T_{\mathrm{P}_{,}, \mathrm{CO}_{2}}(t) \mathrm{d} t} / \frac{\int_{0}^{H} C_{\mathrm{P} x}(t) F_{x} \mathrm{~d} t}{\int_{0}^{H} C_{\mathrm{P}, \mathrm{CO}_{2}}(t) F_{\mathrm{CO}_{2}} \mathrm{~d} t} .
$$

The equivalence between IGTP and GWP can be understood by rewriting the ratio IGTP/GWP in the following way

$$
\frac{\operatorname{IGTP}(H)}{\operatorname{GWP}(H)}=\frac{\int_{0}^{H} T_{\mathrm{P} x}(t) \mathrm{d} t}{\int_{0}^{H} \lambda C_{\mathrm{P} x}(t) F_{X} \mathrm{~d} t} \frac{\int_{0}^{H} \lambda C_{\mathrm{P}, \mathrm{CO}_{2}}(t) F_{\mathrm{CO}_{2}} \mathrm{~d} t}{\int_{0}^{H} T_{\mathrm{P}, \mathrm{CO}_{2}}(t) \mathrm{d} t} .
$$

Equation (4) was obtained by multiplying the expression in Eq. (3) by $\lambda$, the climate sensitivity, in both the numerator and the denominator. When multiplying $C_{\mathrm{P} x}(t) F_{x}$ by $\lambda$ we get the equilibrium temperature response for gas $X$. Thus, the first ratio on the right hand side of Eq. (4) is the integrated (transient) temperature change divided by the integrated equilibrium temperature change for a gas $X$ (following a pulse emissions).

The equilibrium and the transient temperature responses of $\mathrm{CH}_{4}$ are illustrated in Fig. 4 (upper panel). As seen in

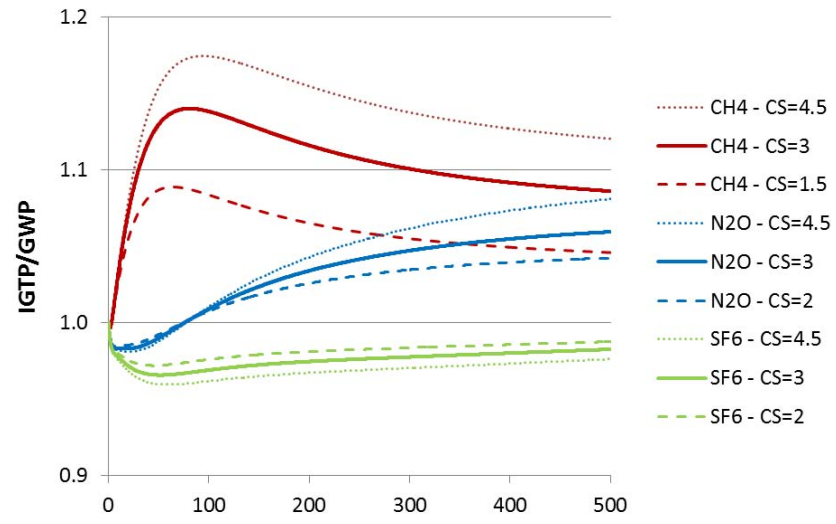

Fig. 3. IGTP/GWP ratio for $\mathrm{CH}_{4}, \mathrm{~N}_{2} \mathrm{O}$ and $\mathrm{SF}_{6}$ depending on climate sensitivity. CS, the climate sensitivity, is given in Kelvins per $\mathrm{CO}_{2}$ equivalent doubling.

Fig. 4, the equilibrium temperature response is higher than the transient response for the first two decades due to heat uptake by the oceans, thereafter the transient temperature response is higher than the equilibrium response due to heat release from the oceans. The integrated equilibrium temperature response (the area under the blue curve) is larger than the integrated transient temperature response (the area under the red curve) for any time horizon $H$, but the areas under the two curves approach the same value asymptotically as $H$ approaches infinity.

This asymptotic behavior can be understood in physical terms; the (integrated) radiative forcing must eventually manifest itself in (integrated) temperature change (see Appendix D for a formal proof of this). Thus, the first ratio on the right hand side in Eq. (4) will be less than one and asymptotically approach one when the time horizon $H$ approaches infinity. Similar arguments hold for the second ratio, as will be discussed below, and for that reason the IGTP to GWP ratio becomes equal to unity as the time horizon approaches infinity, see Appendix D, O'Neill (2000), and Peters et al. (2011).

The second ratio on the right hand side of Eq. (4) is the integrated equilibrium temperature change divided by the integrated temperature change for a pulse emission of $\mathrm{CO}_{2}$ (see lower panel, Fig. 4). Here the same arguments can be used as for $\mathrm{CH}_{4}$ above, although the time scales involved when approaching unity is much longer because of the much longer perturbation life time of $\mathrm{CO}_{2}$. Hence, the second ratio on the right hand side in Eq. (4) will be larger than one and asymptotically approach one as $H$ approaches infinity.

The overall ratio in Eq. (4) will thus become slightly larger than one when $\mathrm{CH}_{4}$ is gas $\mathrm{X}$, since the ratio for $\mathrm{CH}_{4}$ (the first ratio on the right hand side) will reach unity faster than the ratio for $\mathrm{CO}_{2}$ since methane has a much shorter life time. This explains the result shown in Fig. 1.

If, on the other hand, the atmospheric life time of gas $X$ is relatively long, such as for $\mathrm{SF}_{6}$, the first ratio would approach 

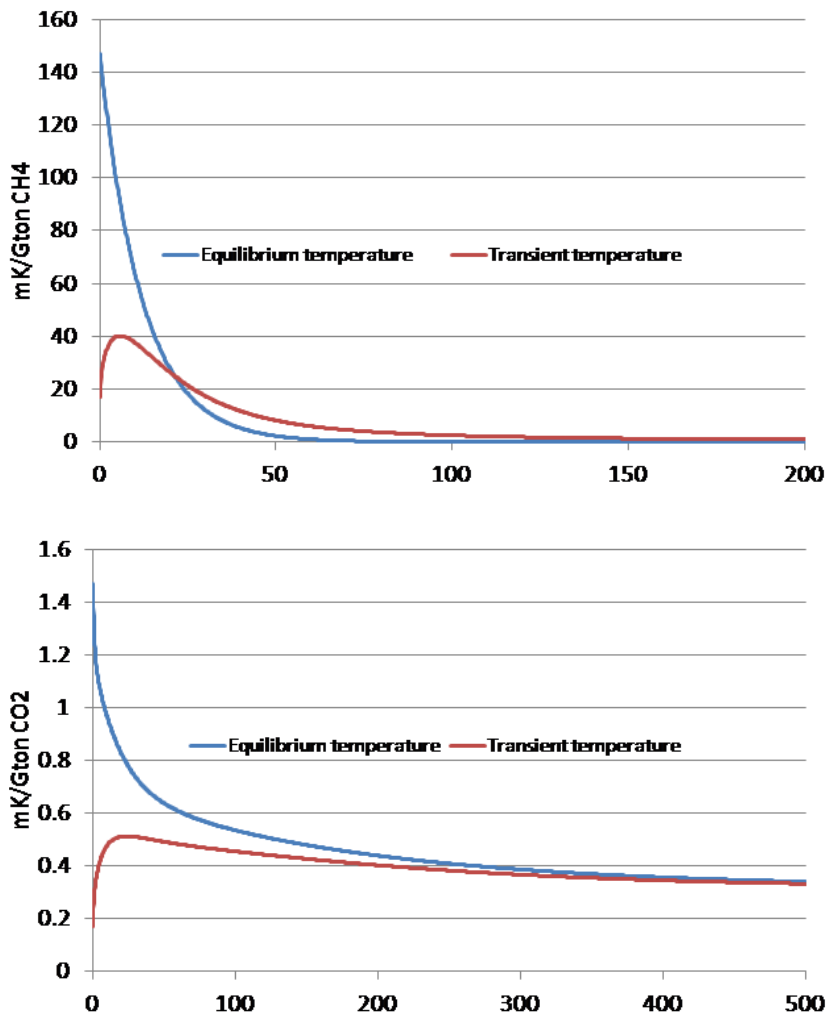

Fig. 4. Equilibrium and transient temperature response for a pulse emission of $\mathrm{CH}_{4}$ (upper panel) and $\mathrm{CO}_{2}$ (lower panel).

unity more slowly than the ratio for $\mathrm{CO}_{2}$. Thus the IGTP-toGWP ratio becomes less than one for $\mathrm{SF}_{6}$. Rotmans and den Elzen (1992) also noted that the ratio IGTP-to-GWP is higher for gases with short life times, although they used slightly different concepts for IGTP and GWP.

For $\mathrm{N}_{2} \mathrm{O}$ the IGTP-to-GWP ratio is initially less than one, but then becomes larger than one. This is explained by the fact that for short time horizons, an emissions pulse of $\mathrm{N}_{2} \mathrm{O}$ decays from the atmosphere more slowly than $\mathrm{CO}_{2}$, but on longer time horizons an emissions pulse of $\mathrm{N}_{2} \mathrm{O}$ decays more rapidly than $\mathrm{CO}_{2}$.

The observation that the IGPT over GWP ratio is lower than one initially, then slightly higher than one, and then eventually returns asymptotically to one for $\mathrm{N}_{2} \mathrm{O}$ is in fact a generic result. It also holds for $\mathrm{CH}_{4}$ (which can be seen if one looks carefully in Fig. 1 for the first few years ${ }^{1}$ ) and $\mathrm{SF}_{6}$ (which would be seen in Fig. 1 if the time horizon had been extended ${ }^{2}$ ), and more generically for all gases with constant decay rates in between those of $\mathrm{CH}_{4}$ and $\mathrm{SF}_{6}$.

\footnotetext{
${ }^{1}$ Since $\mathrm{CO}_{2}$ (approximately) decays with a series of exponential time constants, one of which is much shorter than the decay time constant of methane (around 1.2 versus $12 \mathrm{yr}$ according to the IPCC AR4 estimate), the IGTP-GWP ratio becomes less than unity the first few years.

${ }^{2}$ The ratio becomes higher than unity for time horizons so long as most of $\mathrm{SF}_{6}$ has decayed away (see also Peters et al., 2011). The
}

\section{Explanation of the results in the sensitivity analysis}

If there is no inertia in the climate system, it can be seen right away from Eq. (4) that the IGTP-to-GWP ratio will become equal to one (both ratios in the right hand side of Eq. (4) are equal to unity, since there is no difference between transient and equilibrium temperature response). Now, when considering the inertia of the climate system (that results from the heat capacity of the oceans) the IGTP-to-GWP ratio will deviate from unity. The larger the inertia is (as a result of higher climate sensitivity or higher diffusivity), the more the deviation of the ratio from unity will be. This is the fundamental reason behind the results of the sensitivity analysis presented in Figs. 2 and 3.

\section{Conclusions}

This paper addresses similarities between different metrics to compare greenhouse gases, in particular between GWP and IGTP. A near equivalence between IGTP and GWP is demonstrated. IGTP and GWP are near equivalent in two ways: (1) they are identical if there is no thermal inertia in the climate system; (2) they are asymptotically equal when the time horizon approaches infinity. The values differ by, at most, some $10 \%$ in the cases studied here. Our research corroborates the results by Peters et al. (2011) and Rotmans and den Elzen (1992) who used different modeling approaches.

We also find a rather general relationship between IGTP and GWP. The ratio IGTP to GWP (for a time horizon in the range a decade to several centuries) is slightly higher than unity for gases with relatively short atmospheric life times (say methane), and lower than unity for gases with relatively long atmospheric life times (say $\mathrm{SF}_{6}$ ). However, if one considers time horizons for the analysis that stretch from just a few years to several thousand years, it turns out that both methane and $\mathrm{SF}_{6}$ exhibit similar features. The ratio IGPT to GWP first drops (for both gases to just below unity) and then increase (to at most some $10 \%$ ) above unity, and then approach unity asymptotically. Also, for gases with life times around $100 \mathrm{yr}$, like nitrous oxide, this pattern can be seen in Fig. 3 more clearly. The reason for this "first dive and then emerge above unity" pattern has to do with the fact that the life time of $\mathrm{CO}_{2}$ cannot be captured in a single time constant.

In addition, we also carry out a sensitivity analysis with respect to the climate sensitivity and the heat diffusivity, and found that the difference between IGTP and GWP increases with the inertia in the climate system (higher inertia stems either from a higher climate sensitivity or from higher heat diffusivity).

The near equivalence between IGTP and GWP can be understood in physical terms. Since the integration of the temperature response must be (over infinitely long time

reason for that is a fraction of the increase in atmospheric concentration of $\mathrm{CO}_{2}$ will remain on longer timescales than the $\mathrm{SF}_{6}$ lifetime. 
horizons) proportional to the integrated radiative forcing of a pulse emission, it follows that GWP and IGTP are asymptotically identical. The analysis here has thus focused on how they deviate on shorter time scales. In Appendix A it is also shown that SGTP and IGTP are identical under assumptions about linearity.

Given that these metrics (GWP, SGTP and IGTP) are either equivalent or near equivalent, the exact choice of these metrics is of less importance for abatement decisions. Hence, there is no compelling reason why IGTP or SGTP should be chosen over GWP.

While it matters little for abatement policy whether IGTP, SGTP or GWP is used when making trade-offs, it is more important to decide whether society should use a metric based on time integrated effects such as IGTP and GWP, a snapshot metric as GTP, or metrics where both economics and physical considerations are taken into account (see Manne and Richels, 2001; O'Neill, 2000; Shine, 2009; Azar and Johansson, 2012). Of equal importance is the question of how to choose the time horizon, regardless of the chosen metric. For these questions, value judgments are needed and they can thus not solely be answered by the scientific community.

\section{Appendix A}

Demonstrating the equivalence between sustained global temperature potential (SGTP) and the integrated global temperature change potential (IGTP)

Shine et al. (2005) state that SGTP and GWP are "near equivalent". They show that this near equivalence holds numerically for certain time horizons. However, they also state that "the near equivalence of the GWP and GTP at $_{\mathrm{s}} 100$ years does not guarantee equivalence at other time horizons". (They refer to SGTP as GTP s). $^{\text {) }}$

Here we show that $\operatorname{SGTP}(H)$ and $\operatorname{IGTP}(H)$ are identical metrics under certain conditions that will be defined below.

SGTP is the temperature response of a gas $X$ emitted at a constant (sustained) rate $\left(1 \mathrm{~kg} \mathrm{yr}^{-1}\right)$ divided by the temperature response following sustained emissions of $\mathrm{CO}_{2}$ $\left(1 \mathrm{~kg} \mathrm{yr}^{-1}\right)$. Or more formally,

$\operatorname{SGTP}(H)=\frac{\operatorname{ASGTP}_{x}(H)}{\operatorname{ASGTP}_{\mathrm{CO}_{2}}(H)}$

where $\operatorname{ASGTP}(H)$ is the absolute temperature response at time $H$ following sustained emissions during the period $0<t<H$, and defined as the integrated effect of a the temperature response of a series of pulse emissions, i.e.
$\operatorname{ASGTP}_{x}(H)=\int_{0}^{H} T_{\mathrm{P} x}(H, \tau) \mathrm{d} \tau$.

Here $T_{\mathrm{P} x}(H, \tau)$ is the temperature response at time $H$ from a pulse emission at time $\tau$.

Now, assume that the temperature response is the same regardless of when in time the pulse emission occurs, i.e. we assume that radiative forcing and adjustment time for each additional unit greenhouse gas are constant, which is standard when estimating GWPs, and we also assume linearity in the temperature response model.

If so,

$T_{\mathrm{P} x}(H, \tau)=T_{\mathrm{P} x}(H-\tau)$

We insert expression (Eq. A3 into Eq. A2), and then, through the variable substitution, $t=H-\tau$, ASGTP can be rewritten as

$\operatorname{ASGTP}_{x}(H)=\int_{0}^{H} T_{\mathrm{P} x}(H-\tau) \mathrm{d} \tau=\int_{0}^{H} T_{\mathrm{P} x}(t) \mathrm{d} t$.

Thus, $\operatorname{ASGTP}_{x}(H)$ is equal to the integrated temperature response from a pulse emission of gas $X$. Since it holds for gas $X$, it will hold for all gases (including $\mathrm{CO}_{2}$ ). Thus, it follows that

$\operatorname{SGTP}(H)=\frac{\operatorname{ASGTP}_{x}(H)}{\operatorname{ASGTP}_{\mathrm{CO}_{2}}(H)}=\frac{\int_{0}^{H} T_{\mathrm{P} x}(t) \mathrm{d} t}{\int_{0}^{H} T_{\mathrm{P}_{,} \mathrm{CO}_{2}}(t) \mathrm{d} t}=\operatorname{IGTP}(H)$.

Thus, SGTP is identical to IGTP when radiative forcing and adjustment time for each additional unit emission of greenhouse gas are constant and linearity holds for the temperature response to radiative forcing changes. Thus, the results reported in this paper for the relationship between IGTP and GWP also hold for SGTP (given these linearity assumptions), i.e. SGTP is "near equivalent" to GWP for reasons explained in Sect. 4 of this paper.

Hence, that SGTP and IGTP are equal measures might seem surprising given that SGTP is an end-point measure, whereas the IGTP is an integrative measure but it follows from the fact that SGTP is a measure of the temperature change at one point in time from a sustained emission, i.e. it is the integrated temperature effect of a series of pulse emissions.

If the background concentrations of $\mathrm{CO}_{2}$ and gas $X$ are changing, SGTP and IGTP will only be approximately equal. 


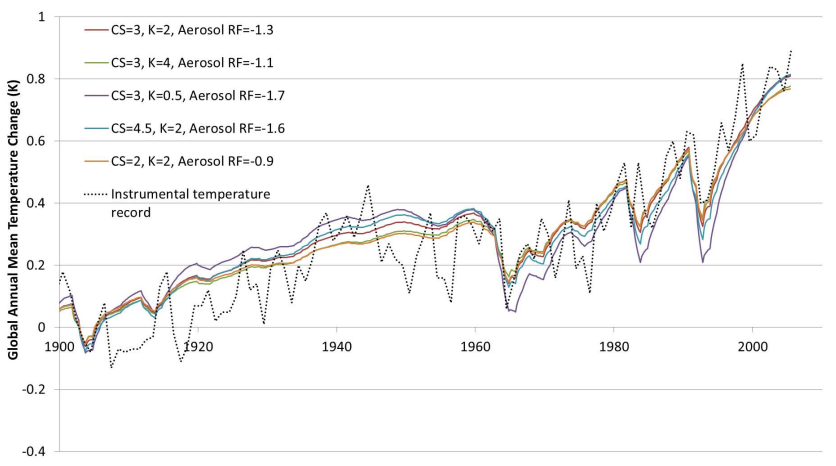

Fig. B1. Modeled and observed historic global mean surface temperature change. The historic temperature series is taken from NASA GISS (2012). $\mathrm{CS}=$ climate sensitivity (Kelvins per $\mathrm{CO}_{2}$ equivalent doubling), $K=$ the vertical heat diffusivity $\left(\mathrm{cm}^{2} \mathrm{~s}^{-1}\right)$. The aerosol forcing is given in $\mathrm{W} \mathrm{m}^{-2}$ for the year 2005 . Historic estimates for the aerosol forcing are scaled linearly with this value.

Table C1. GWP and IGTP values for $\mathrm{CH}_{4} . K$, the vertical heat diffusivity, is given in $\mathrm{cm}^{2} \mathrm{~s}^{-1}$ and $\mathrm{CS}$, the climate sensitivity, is given in Kelvins per $\mathrm{CO}_{2}$ equivalent doubling.

\begin{tabular}{lrrrrrr}
\hline Time & GWP & \multicolumn{5}{c}{ IGTP } \\
\cline { 2 - 7 } horizon & & $\mathrm{CS}=3$ & $\mathrm{CS}=4.5$ & $\mathrm{CS}=2$ & $\mathrm{CS}=3$ & $\mathrm{CS}=3$ \\
& & $K=2$ & $K=2$ & $K=2$ & $K=4$ & $K=0.5$ \\
\hline 20 & 72 & 77 & 78 & 76 & 77 & 76 \\
100 & 25 & 29 & 30 & 27 & 29 & 28 \\
500 & 8 & 8 & 9 & 8 & 9 & 8 \\
\hline
\end{tabular}

\section{Appendix B}

\section{Modeling historic temperatures}

Our UDEBM was run using historic radiative forcing data from the representative concentration pathway scenarios (see Meinshausen et al., 2011b) using different combinations of values for the climate sensitivity (CS), the vertical heat diffusivity $(K)$ and the total radiative forcing from aerosols. It can be seen in Fig. B1 that the model fairly well reproduces the historic global mean surface temperature change over the period 1900-2005 (as estimated by NASA GISS, 2012) for each set of parameter combinations. When changing either the climate sensitivity or the vertical heat diffusivity, changes in the aerosol forcing are required to maintain a good fit with historic temperatures. There is significant uncertainty in the aerosol forcing, but our assumptions are well within the estimated range (Forster et al., 2007).

\section{Appendix C}

\section{IGTP and GWP values}

In Tables C1-C3, we summarize the GWP and IGTP values for $\mathrm{CH}_{4}, \mathrm{~N}_{2} \mathrm{O}$ and $\mathrm{SF}_{6}$ for different assumptions on the climate sensitivity and effective vertical heat diffusivity.
Table C2. GWP and IGTP values for $\mathrm{N}_{2} \mathrm{O} . K$, the vertical heat diffusivity, is given in $\mathrm{cm}^{2} \mathrm{~s}^{-1}$ and CS, the climate sensitivity, is given in Kelvins per $\mathrm{CO}_{2}$ equivalent doubling.

\begin{tabular}{lcccccc}
\hline Time & GWP & \multicolumn{5}{c}{ IGTP } \\
\cline { 3 - 7 } horizon & & $\mathrm{CS}=3$ & $\mathrm{CS}=4.5$ & $\mathrm{CS}=2$ & $\mathrm{CS}=3$ & $\mathrm{CS}=3$ \\
& & $K=2$ & $K=2$ & $K=2$ & $K=4$ & $K=0.5$ \\
\hline 20 & 289 & 284 & 283 & 285 & 284 & 284 \\
100 & 298 & 301 & 301 & 300 & 301 & 300 \\
500 & 153 & 162 & 166 & 160 & 165 & 158 \\
\hline
\end{tabular}

Table C3. GWP and IGTP values for $\mathrm{SF}_{6} . K$, the vertical heat diffusivity, is given in $\mathrm{cm}^{2} \mathrm{~s}^{-1}$ and $\mathrm{CS}$, the climate sensitivity, is given in Kelvins per $\mathrm{CO}_{2}$ equivalent doubling.

\begin{tabular}{lcccccc}
\hline Time & GWP & \multicolumn{5}{c}{ IGTP } \\
\cline { 3 - 7 } horizon & & CS $=3$ & CS $=4.5$ & CS $=2$ & CS $=3$ & CS $=3$ \\
& & $K=2$ & $K=2$ & $K=2$ & $K=4$ & $K=0.5$ \\
\hline 20 & 16200 & 15800 & 15700 & 15800 & 15800 & 15800 \\
100 & 22800 & 22100 & 21900 & 22300 & 22000 & 22300 \\
500 & 32600 & 32000 & 31800 & 32200 & 31900 & 32300 \\
\hline
\end{tabular}

\section{Appendix D}

\section{Demonstrating the asymptotic equivalence between IGTP and GWP}

Let us assume the following simple model of the climate system with thermal inertia:

$C \frac{\mathrm{d} T}{\mathrm{~d} t}=F(t)-T / \lambda$

where $C$ is the thermal inertia of the layer that should be heated, $F(t)$ the radiative forcing, $T$ the increase in temperature and $\lambda$ the climate sensitivity (as above). Assume now that one $\mathrm{kg}$ of gas $X$ is emitted, and that it decays exponentially with a life time of $\tau_{x}, F_{x}(t)=F_{x} e^{-t / \tau_{x}}$. We then obtain the temperature response to a pulse emission of gas $X$ as

$T_{\mathrm{P} x}(t)=\frac{\tau_{x} \lambda F_{x}}{\tau_{x}-\lambda C}\left(e^{-t / \tau_{x}}-e^{-t / \lambda C}\right)$.

Integrating over the temperature response gives

$$
\begin{aligned}
\int_{0}^{H} T_{\mathrm{P} x}(t) \mathrm{d} t & =\frac{\tau_{x} \lambda F_{x}}{\tau_{x}-\lambda C}\left\{\tau_{x}\left(1-e^{-H / \tau_{x}}\right)\right. \\
& \left.-\lambda C\left(1-e^{-H / \lambda C}\right)\right\} .
\end{aligned}
$$

We notice that if $H$ goes to infinity, the integral converges towards $\tau_{x} \lambda F_{x}$. This can be understood in the following way: since the integral over the temperature response is the same as the absolute SGTP for a gas $X$, we may instead of integrating the temperature response, directly consider the equilibrium temperature response of a sustained pulse of gas $X$. This 
temperature response is equal to the equilibrium increase in the atmospheric mass of gas $X$ multiplied by the forcing per $\mathrm{kg}$ multiplied by the climate sensitivity, i.e. $\tau_{x} \lambda F_{x}$.

For $\mathrm{CO}_{2}$, the atmospheric "decay" function is somewhat more complicated. We assume that it can be approximated by a sum of exponential functions and a constant term (see Forster et al., 2007), so that the radiative forcing at time $t$ from the emission of $\mathrm{kg}$ of $\mathrm{CO}_{2}$ at time 0 is equal to

$F_{\mathrm{CO}_{2}}(t)=F_{\mathrm{CO}_{2}}\left(\alpha_{0}+\sum_{i=1}^{3} \alpha_{i} e^{-t / \tau_{\mathrm{CO}_{2}, i}}\right)$.

If so, the temperature following a pulse emission would be

$$
\begin{aligned}
T_{\mathrm{P}, \mathrm{CO}_{2}}(t) & =\lambda F_{\mathrm{CO}_{2}}\left\{\alpha_{0}\left(1-e^{-\frac{t}{\lambda C}}\right)\right. \\
& \left.+\sum_{i=1}^{3} \frac{\alpha_{i} \tau_{i}}{\tau_{i}-\lambda C}\left(e^{-\frac{t}{\tau_{i}}}-e^{-\frac{t}{\lambda C}}\right)\right\} .
\end{aligned}
$$

Integrating over the temperature response gives

$$
\begin{aligned}
& \int_{0}^{H} T_{\mathrm{P}, \mathrm{CO}_{2}}(t) \mathrm{d} t=\lambda F_{\mathrm{CO}_{2}}\left\{\alpha_{0}\left(H+C \lambda\left(e^{-\frac{H}{\lambda C}}-1\right)\right)\right. \\
& \left.+\sum_{i=1}^{3} \frac{\alpha_{i} \tau_{i}}{\tau_{i}-\lambda C}\left\{\tau_{i}\left(1-e^{-\frac{H}{\tau_{i}}}\right)-\lambda C\left(1-e^{-\frac{H}{\lambda C}}\right)\right\}\right\} .
\end{aligned}
$$

Now calculate IGTP/GWP in the limit $H->\infty$

$$
\begin{aligned}
& \lim \operatorname{IGTP}(H) \\
& H \rightarrow \infty \overline{\operatorname{GWP}(H)} \\
& =\lim _{H \rightarrow \infty} \frac{\frac{\frac{\tau_{x} \lambda F_{x}}{\tau_{x}-\lambda C}\left\{\tau_{x}\left(1-e^{-\frac{H}{\tau_{x}}}\right)-\lambda C\left(1-e^{-\frac{H}{\lambda C}}\right)\right\}}{\lambda F_{\mathrm{CO}_{2}}\left\{\alpha_{0}\left(H+C \lambda\left(e^{-\frac{H}{\lambda C}}-1\right)\right)+\sum_{i=1}^{3} \frac{\alpha_{i} \tau_{i}}{\tau_{i}-\lambda C}\left\{\tau_{i}\left(1-e^{-\frac{H}{\tau_{i}}}\right)-\lambda C\left(1-e^{-\frac{H}{\lambda C}}\right)\right\}\right\}}}{\frac{\tau_{x} F_{x}\left(1-e^{-\frac{H}{\tau_{x}}}\right)}{F_{\mathrm{CO}_{2}}\left\{\alpha_{0} H+\sum_{i=1}^{3} \alpha_{i} \tau_{i}\left(1-e^{-\frac{H}{\tau_{i}}}\right)\right\}}}=1 \text { (D7) }
\end{aligned}
$$

Acknowledgements. Financial support from the Swedish Energy Agency and Carl Bennet AB is gratefully acknowledged.

Edited by: K. Keller

\section{References}

Azar, C. and Johansson, D. J. A.: Valuing the non- $\mathrm{CO}_{2}$ climate impacts of aviation, Climatic Change, 111, 559-579, doi:10.1007/s10584-011-0168-8, 2012.

Baker, M. B. and Roe, G. H.: The shape of things to come: why is climate change so predictable?, J. Climate, 22, 4574-4589, 2009.

Fisher, D. A., Hales, C. H., Wang, W.-C., Ko, M. K. W., and Sze, N. D.: Model calculation on the relative effects of CFCs and their replacements on global warming, Nature, 344, 513-516, 1990.
Forster, P., Ramaswamy, V., Artaxo, P., Berntsen, T., Betts, R., Fahey, D. W., Haywood, J., Lean, J., Lowe, D. C., Myhre, G., Nganga, J., Prinn, R., Raga, G., Schulz, M., and Van Dorland, R.: Changes in Atmospheric Constituents and in Radiative Forcing, in: Climate Change 2007: The Physical Science Basis. Contribution of Working Group I to the Fourth Assessment Report of the Intergovernmental Panel on Climate Change, edited by: Solomon, S., Qin, D., Manning, M., Chen, Z., Marquis, M., Averyt, K. B., Tignor, M., and Miller, H. L., Cambridge University Press, Cambridge, UK and New York, NY, USA, 2007.

Gillet, X. and Matthews, H. D.: Accounting for carbon cycle feedbacks in a comparison of the global warming effects of greenhouse gases, Environ. Res. Lett., 5, 034011, doi:10.1088/17489326/5/3/034011, 2010.

Hansen, J., Russell, G., Lacis, A., Fung, I., Rind, D., and Stone, P.: Climate Response Times: Dependence on Climate Sensitivity and Ocean Mixing, Science, 229, 857-859, 1985.

Hansen, J., Sato, M., Ruedy, R., Nazarenko, L., Lacis, A., Schmidt, G. A., Russell, G., Aleinov, I., Bauer, M., Bauer, S., Bell, N., Cairns, B., Canuto, V., Chandler, M., Cheng, Y., Del Genio, A., Faluvegi, G., Fleming, E., Friend, A., Hall, T., Jackman, C., Kelley, M., Kiang, N., Koch, D., Lean, J., Lerner, J., Lo, K., Menon, S., Miller, R., Minnis, P., Novakov, T., Oinas, V., Perlwitz, Ja., Perlwitz, Ju., Rind, D., Romanou, A., Shindell, D., Stone, P., Sun, S., Tausnev, N., Thresher, D., Wielicki, B., Wong, T., Yao, M., and Zhang, S.: Efficacy of Climate Forcings, J. Geophys. Res., 110, D18104, doi:10.1029/2005JD005776, 2005.

IPCC:Report of the IPCC Expert meeting on the Science of Alternative Metrics 18-20 March 2009, Oslo, available at http://www.ipcc.ch/pdf/supporting-material/ expert-meeting-metrics-oslo.pdf (last access: 5 February 2012), 2009.

Johansson, D. J. A.: Temperature stabilization, ocean heat uptake and radiative forcing overshoot profiles, Climatic Change, 108, 107-134, 2011.

Johansson, D. J. A.: Economics- and Physical-Based Metrics for Comparing Greenhouse Gases, Climatic Change, 110, 101-121, 2012.

Lashof, D. A. and Ahuja, D. R.: Relative contributions of greenhouse gas emissions to global warming, Nature, 344, 529-531, 1990.

Manne, A. S. and Richels, R. G.: An alternative approach to establishing trade-offs among greenhouse gases, Nature, 410, 675677, 2001.

Meinshausen, M., Raper, S. C. B., and Wigley, T. M. L.: Emulating coupled atmosphere-ocean and carbon cycle models with a simpler model, MAGICC6 - Part 1: Model description and calibration, Atmos. Chem. Phys., 11, 1417-1456, doi:10.5194/acp11-1417-2011, 2011a.

Meinshausen, M., Smith, S. J., Calvin, K. V., Daniel, J. S. Kainuma, M. L. T., Lamarque, J.-F., Matsumoto, K., Montzka, S. A., Raper, S. C. B., Riahi, K., Thomson, A. M., Velders, G. J. M., and van Vuuren, D.: The RCP Greenhouse Gas Concentrations and their Extension from 1765 to 2300, Climatic Change, 109, 213-241, 2011b.

NASA GISS: GISS surface temperature analysis, available at http: //data.giss.nasa.gov/gistemp/, last access: 8 January 2012. 
Olivié, D. and Stuber, N.: Emulating AOGCM results using simple climate models, Clim. Dynam., 35, 1257-1287, 2010.

O'Neill, B. C.: The jury is still out on global warming potentials, Climatic Change, 44, 427-443, 2000.

Peters, G. P., Aamaas, B., Berntsen, T., and Fuglestvedt, J. S.: The integrated global temperature change potential (iGTP) and relationships between emission metrics, Environ. Res. Lett., 6, 044021, doi:10.1088/1748-9326/6/4/044021, 2011.

Raper, S. C. B., Gregory, J. M., and Osborn, T. J.: Use of an upwelling-diffusion energy balance climate model to simulate and diagnose A/OGCM results, Clim. Dynam., 17, 601-613, 2001.

Rodhe, H.: A comparison of the contribution of various gases to the greenhouse effect, Science, 248, 1217-1219, 1990.

Rotmans, J. and den Elzen, M. G. J.: A model-based approach to the calculation of global warming potentials (GWP), Int. J. Climatol., 12, 865-874, 1992.

Sarofim, M. C.: The GTP of methane: modeling analysis of temperature impacts of methane and carbon dioxide reductions, Environ. Model. Assess., 17, 231-239, 2012.
Shine, K. P.: The global warming potential, The need for an interdisciplinary retrial, Climatic Change, 96, 467-472, 2009.

Shine, K. P., Derwent, R. G., Wuebbles, D. J., and Morcrette, J. J.: Radiative forcing of climate, in: Climate change: the IPCC scientific assessment, edited by: Houghton, J. T., Jenkins, G. J., and Ephraums, J. J., Cambridge University Press, Cambridge, 1990.

Shine, K. P., Fuglestvedt, J. S., Hailemariam, K., and Stuber, N.: Alternatives to the global warming potential for comparing climate impacts of emissions of greenhouse gases, Climatic Change, 68, 281-302, 2005.

Solomon, S., Qin, D., Manning, M., Chen, Z., Marquis, M., Averyt, K. B., Tignor, M., and Miller, H. L.: Climate Change 2007: The Physical Science Basis, Contribution of Working Group I to the Fourth Assessment Report of the Intergovernmental Panel on Climate Change, Cambridge University Press, Cambridge, UK and New York, NY, USA, 996 pp., 2007. 